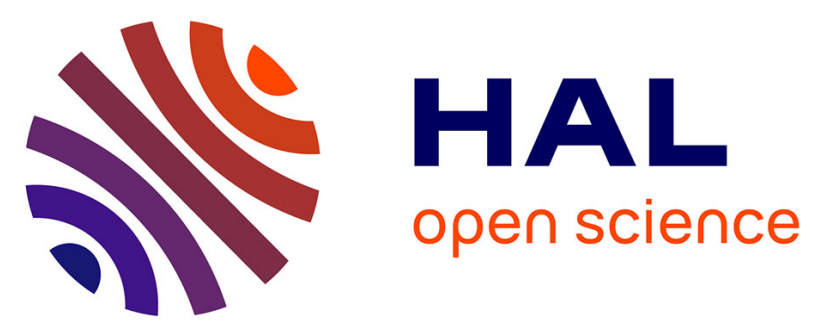

\title{
Electromagnetic characterization of soil-litter media: application to the simulation of the microwave emissivity of the ground surface in forests
}

François Demontoux, Jennifer Grant, Heather Lawrence, Bénédicte Le Crom, Gilles Ruffié, Jean Pierre Wigneron, Valery Mironov

\section{To cite this version:}

François Demontoux, Jennifer Grant, Heather Lawrence, Bénédicte Le Crom, Gilles Ruffié, et al.. Electromagnetic characterization of soil-litter media: application to the simulation of the microwave emissivity of the ground surface in forests. European Physical Journal: Applied Physics, 2008, 44 (44), pp.303-315. 10.1051/epjap:2008168 . hal-00345723

\section{HAL Id: hal-00345723 \\ https://hal.science/hal-00345723}

Submitted on 31 May 2020

HAL is a multi-disciplinary open access archive for the deposit and dissemination of scientific research documents, whether they are published or not. The documents may come from teaching and research institutions in France or abroad, or from public or private research centers.
L'archive ouverte pluridisciplinaire HAL, est destinée au dépôt et à la diffusion de documents scientifiques de niveau recherche, publiés ou non, émanant des établissements d'enseignement et de recherche français ou étrangers, des laboratoires publics ou privés.

$$
\text { Copyright }
$$




\title{
Electromagnetic characterization of soil-litter media: application to the simulation of the microwave emissivity of the ground surface in forests
}

\author{
F. Demontoux ${ }^{1, a}$, B. Le Crom ${ }^{1,2}$, G. Ruffié ${ }^{1}$, J.P. Wigneron ${ }^{2}$, J.P. Grant ${ }^{2,3}$, V.L. Mironov ${ }^{4}$, and H. Lawrence ${ }^{1,2}$ \\ 1 Université Bordeaux 1, Laboratoire IMS-UMR 5218, 16 avenue Pey-Berland, 33607 Pessac, France \\ 2 INRA-Unité de Bioclimatologie, BP 81, 33883 Villenave d'Ornon Cedex, France \\ 3 Faculty of Earth and Life Sciences, Vrije Universiteit Amsterdam, 1085 De Boelelaan, Holland \\ 4 Radiophysics of Remote Sensing Laboratory, Kirensky Institute of Physics, Siberian Branch, \\ Russian Academy of Sciences, Russian
}

Received: 29 March 2007 / Received in final form: 3 December 2007 / Accepted: 25 August 2008 Published online: 6 December 2008 - (C) EDP Sciences

\begin{abstract}
In order to improve our knowledge of the emitted signal of forests at $L$-band $(1.4 \mathrm{GHz})$ we focused this study on permittivity measurements of heterogenic natural media such as soil or litter consisting of plant debris and organic matter. This study was done in the context of the upcoming SMOS (Soil Moisture and Ocean Salinity) satellite mission that will attempt to map surface soil moisture from $L$-band $(1.4 \mathrm{GHz})$ passive microwave measurements. In the field of passive microwaves, very little information exists about the behaviour of the $L$-band signal of forests especially when litter is included in the soil-vegetation system. To date very few analyses have investigated the dielectric behaviour of the litter layer and its influence on the microwave emission of forests is generally neglected.
\end{abstract}

PACS. 06.20.Dk Measurement and error theory - 77.22.-d Dielectric properties of solids and liquids 93.90.+y Other topics in geophysical observations, instrumentation, and techniques (restricted to new topics in section 93 )

This paper describes a technique used to measure the permittivity of soil and litter with a rectangular waveguide taking into account the heterogeneity of the media. The study was based on soil and litter samples collected in the coniferous forest of Les Landes, near Bordeaux, France. Particular attention was given to the impact of measurement errors on the calculation of permittivity. The measurements of the soil and litter dielectric constants were then used in a numerical model to simulate the microwave emissivity of the soil-litter system. As an example, it is shown that error in measurements lead to variations in the emissivity calculations of up to 0.025 at maximum (for an emissivity of $0.73, S M=30 \%$ ), which equals a total error of $3.36 \%$ of the calculated soil-litter emissivity.

It was found that variations in the litter dielectric constant and layer thickness have a strong influence on the calculation of forest ground emissivity. As an example a $3 \mathrm{~cm}$ litter thickness can lead to a change of $\sim 0.15$ between the soil and the soil-litter system emissivity (which equals an increase of about $20.4 \%$ in terms of surface emissivity).

\section{Introduction}

The SMOS (Soil Moisture and Ocean Salinity) mission, which is scheduled for launch in 2008, is the second Earth

\footnotetext{
a e-mail: francois.demontoux@ims-bordeaux.fr
}

Explorer mission to be developed as part of ESA's Living Planet Program. The project is a collaboration between three European Agencies: the European Space Agency (ESA) [1], the French National Centre of Space Studies (CNES) [2] and the Spanish Centre for Technical and Industrial Development (CDTI). SMOS will carry the firstever polar orbiting 2-D interferometric radiometer which will provide spatial and temporal maps of surface soil moisture over land surfaces and salinity over the oceans. The data acquired from the mission will contribute to improve our knowledge of the Earth's water cycle. The applications of SMOS data are numerous: better weather and extreme-event forecasting, a contribution to seasonalclimate forecasting, observations over regions of ice and snow, etc. $[3,4]$.

Over the land surfaces, it comes as no surprise that the amount of water in the soil has an influence on climate variables, as soil moisture is a key variable in the exchange of water and thermal energy between the land surface and the atmosphere.

The maps of soil moisture and ocean salinity will be derived from the SMOS measurements of the emitted microwave radiation around the frequency of $1.4 \mathrm{GHz}$ ( $L$-band). Over land surfaces, the microwave emission responds to change in the dielectric constant of materials, which is mainly a result of variation in their water content. 
The SMOS pixel is approximately $40 \mathrm{~km} \times 40 \mathrm{~km}$ and one image obtained by the radiometer will generally include a mixture of various cover types. In particular, it will often include forests since a large fraction of the land surface is covered by this vegetation type. Very few experimental campaigns over forests have been carried out to date and few studies have investigated their microwave signatures [5,24]. Furthermore, in many temperate and boreal forests, a distinct litter layer (consisting of plant debris and organic matter) is present between the soil and the vegetation. Over low vegetation covers such as grass, it was found that this layer has strong effects. It attenuates the soil emission and has a strong self-emission. The presence of this layer generally leads to an important increase in the surface microwave emission $[6-8,25]$. The effect of this layer has until now generally been neglected in the computation of soil microwave emission and very few experimental data sets are available to validate modelling approaches such as that used for SMOS $[9,10]$.

This study aims at investigating a method for measuring the complex permittivity of soil and litter. Tests were done using samples of soil and litter taken from a coniferous forest stand in the Les Landes forest (near Bordeaux, France). These dielectric properties were then used in a multilayer ground model simulating the microwave emission of the soil-litter medium. The objective was to analyse the influence of the litter characteristics - in terms of layer thickness $(0-10 \mathrm{~cm})$ and moisture content $(0-37.5 \%$ for the soil moisture) on the surface emission.

This study presents the technique used for measuring the dielectric properties of the soil and litter samples. The measurements were compared with simulation of a dielectric mixing model and ranges of permittivity were defined in order to take into account the errors of measurement or the heterogeneity of the considered medium. Next, a numerical model which was developed in order to calculate the emissivity of a multilayer system is presented. This model was used to evaluate the effect of the litter layer. More specifically, the impact of errors made in the measurements of soil-litter dielectric properties on the computed soil-litter emissivity was evaluated.

\section{Material and methods}

\subsection{Measurements}

Changes in the dielectric constant of the soil or the litter, which are mainly due to variations in water content, produce a variation in the soil-litter system emission. Knowledge of the electromagnetic properties of these two materials is thus fundamental when computing the system emissivity.

The objective of this study was to estimate an equivalent permittivity of soil and litter which are strongly heterogeneous mediums. A modelling approach using a dielectric mixing method (e.g. [17]) could have been considered. However, measurements on samples of sufficient size $\left(83.85 \mathrm{~mm}^{3}\right.$ and $\left.167.7 \mathrm{~mm}^{3}\right)$ make it possible to obtain a
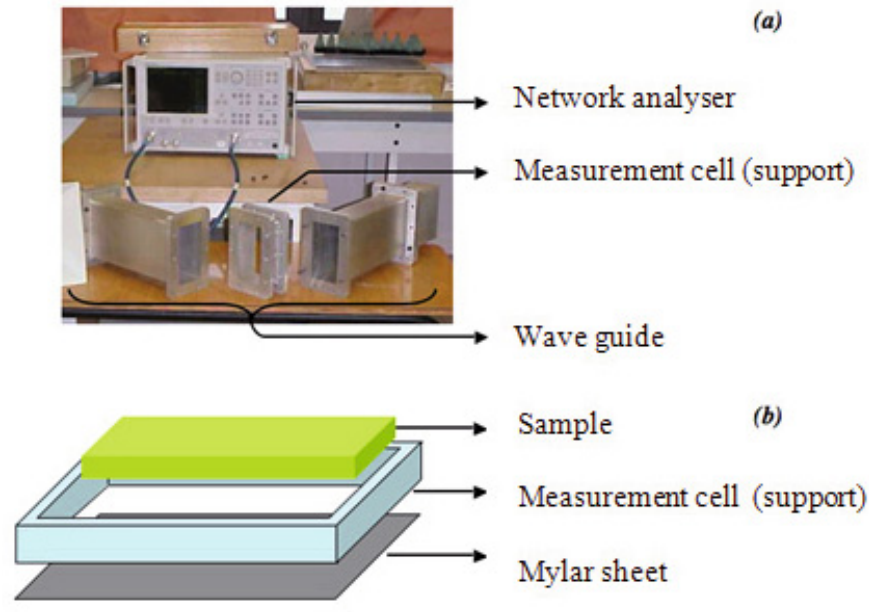

Fig. 1. (Color online) Measurement instruments (a) with details of the measurement cell (b).

direct estimate of the influence of actual heterogeneities on the equivalent permittivity of the medium.

\subsubsection{Measurements with a wave guide technique}

All dielectric measurements presented in this study were done using a wave-guide technique (see Fig. 1) in an airconditioned room $\left(22{ }^{\circ} \mathrm{C}+/-1{ }^{\circ} \mathrm{C}\right)$ and using the frequency of the SMOS mission radiometer $(1.4 \mathrm{GHz})$. This method enabled us to work on samples large enough to account for the layer heterogeneity, in order to measure the effective permittivity of the soil and litter media. The samples were inserted inside a section of the wave guide which was used as a support containing the sample (Fig. 1). At the bottom of this wave guide section, a sheet $(100 \mu \mathrm{m}$ thick Mylar sheet), was used to hold the sample in place. This sheet was considered to be quasi-transparent for the electromagnetic waves. According to rough estimates of the permittivity values of each material, and of the attenuation of the wave, two kinds of support were used:

- a $1 \mathrm{~cm}$ thick support for the soil (high permittivity);

- a $2 \mathrm{~cm}$ thick support for the litter (weak permittivity).

Manual pressure was applied to the sample to reproduce as much as possible its density in real conditions.

The electromagnetic parameters of the samples were determined using the Nicolson, Ross and Weir method (NRW) [12] for rectangular waveguides. This calculation process is based on reflection and transmission measurements and the network analyzer is well suited for this type of measurement. The principle of the calculation is based on the fact that introduction of the sample into the guide produces a change of impedance. The waveguide is a two ports system (each port is an input and an output of the system) in which multiple reflections within the material are taken into account by the coefficients $S_{11}$ and $S_{21}$. These latter are the coefficients of the scattering matrix which represents the various exchange coefficients between all the inputs and outputs of the system. This makes it 
possible to calculate $\Gamma(|\Gamma| \leqslant 1)$, the coefficient of the first reflection and $T$, the coefficient of transmission, using the following equations $[13,14]$ :

$$
\begin{aligned}
& \Gamma=K \pm \sqrt{K^{2}-1} \\
& T=\frac{S_{11}+S_{21}-\Gamma}{1-\left(S_{11}+S_{21}\right) \Gamma} \\
& K=\frac{S_{11}^{2}-S_{21}^{2}+1}{2 S_{11}} .
\end{aligned}
$$

Because it concerns a non magnetic sample, its electromagnetic characteristics are given by:

$$
\left\{\begin{array}{l}
\mu^{*}=1 \\
\varepsilon^{*}=\varepsilon^{\prime}-j \varepsilon^{\prime \prime}=\frac{\gamma_{g} \lambda_{0 g}}{j 2 \pi} \frac{1-\Gamma}{1+\Gamma}
\end{array}\right.
$$

where:

$$
\gamma_{g}=\frac{-1}{2 d} \ln (T)=\frac{-1}{2 d}(\ln (|T|)+j \phi)
$$

and:

$$
\lambda_{0 g}=\frac{1}{\sqrt{\frac{1}{\lambda_{0}^{2}}-\frac{1}{\lambda_{c}^{2}}}}
$$

with: $\gamma_{g}$ guided propagation constant in the rectangular waveguide filled with the sample; $\lambda_{0 g}(\mathrm{~mm})$ wavelength in the empty guide; $\lambda_{0}(\mathrm{~mm})$ vacuum wavelength; $\lambda_{c}(\mathrm{~mm})$ rectangular guide cut-off wavelength; $\lambda_{c}=2 b(\mathrm{~mm})$ where $b$ is the characteristic waveguide width. For $1.4 \mathrm{GHz}$ band the waveguide dimensions are: height $a=65 \mathrm{~mm}$; width $b=130 \mathrm{~mm} ; d(\mathrm{~mm})$ sample thickness.

From the above equations (Eqs. (1-6)), the average values of the real and imaginary parts of the dielectric constant, $\varepsilon^{\prime}$ and $\varepsilon^{\prime \prime}$ respectively, are computable from measurements of the coefficients $S_{11}$ and $S_{21}$. These computations were made in the $1.3-1.5 \mathrm{GHz}$ measurement domain considered in the experimental setup.

In this study, the measurements of the complex coefficients $S_{11}$ and $S_{21}$ of the scattering matrix were done with a network analyzer ANRITSU 37325A [18] which was controlled by a computer for the acquisition and processing of the data. The measurement process began with the measurement of the $S_{21}$ coefficient from the empty wave guide followed by a short-circuit measurement of the $S_{11}$ coefficient in the support plan, in order to define a reference plane to calibrate the system. Next the dry sample was weighed, and then placed in the support of the waveguide. For each measurement, a given quantity of distilled water was sprayed over the sample using a syringe, then after a certain time (so as to allow for the diffusion of water in the sample) the measurements of $S_{11}$ and $S_{21}$ were made. At the end of the measurements the sample was weighed again, so as to make an additional verification of the total quantity of water injected in the sample.

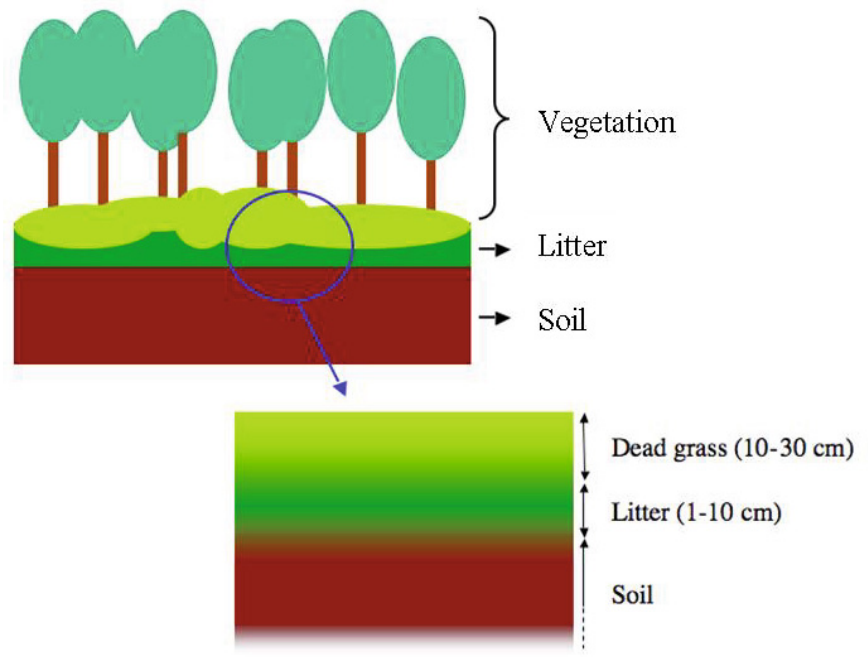

Fig. 2. (Color online) In situ configuration of the collected samples.

\subsubsection{Sample preparation}

The measurements presented in this study were done using soil and litter samples collected on February 15th, 2006 at the INRA site of Bray in the coniferous forest of Les Landes (southwest France). A geometric representation of the soil-litter system over the site is given in Figure 2. In certain cases, the soil-litter system can be overlain by a layer of dead grass (mainly Molinia caerulea L. Moench). In this study, we focus our investigations on the effect of the litter layer, and the dead grass layer will not be considered.

Before carrying out the dielectric measurements at $1.4 \mathrm{GHz}$, the soil and litter samples were dried for one day $(24 \mathrm{~h})$ at $105{ }^{\circ} \mathrm{C}$ for soil and for three days $(72 \mathrm{~h})$ at $65{ }^{\circ} \mathrm{C}$ for litter. These protocols were specifically designed not to denature the soil or litter material [11,17]. During the measurement process, the samples were gradually moistened and the change in weight due to the increase in moisture content was monitored continuously.

In this study the classical calculation methods of soil and litter moisture content were used [11]. Volumetric soil $(S M)$ and gravimetric litter $(L M)$ moisture content were defined by:

$$
\begin{aligned}
& S M=\frac{W_{W}^{S}}{W_{D r y}^{S}} \rho_{b} \\
& L M=\frac{W_{W}^{L}}{W_{D r y}^{L}+W_{W}^{L}}
\end{aligned}
$$

where $W_{W}^{I}$ is the water weight in the sample (g) with $I=S$ for soil and $I=L$ for litter; $W_{D r y}^{I}$ is the weight of the dry sample $(\mathrm{g})$ and $\rho_{b}$ is the soil bulk density $\left(\mathrm{g} / \mathrm{cm}^{3}\right)$.

\subsection{Modelling}

The emissivity $e$ of an object is the ratio of energy radiated by this object to the energy radiated by a black body of the 
same temperature. It is a measure of a material's ability to absorb and radiate energy. This emissivity depends on configuration parameters such as polarization, wavelength and emission angle. According to the Rayleigh-Jeans approximation, emissivity in the microwave region may be expressed as [11]:

$$
e_{S}=\frac{T_{B}}{T_{S}}
$$

where $e_{S}$ is the surface emissivity, $T_{B}$ is the brightness temperature and $T_{S}$ is the physical temperature of the surface. $T_{B}$ can be measured with a microwave radiometer which is a passive and highly sensitive microwave sensor [15].

Soil emissivity is related to reflectivity through the equation:

$$
e_{S}=1-\Gamma_{S}
$$

where $\Gamma_{S}$ is the reflectivity of the soil which depends mainly on soil permittivity and roughness.

\subsubsection{Microwave models}

In this study, two models were used to simulate the microwave emissivity of the soil-litter medium in the Les Landes forest:

(i) a simple radiative transfer model considering a threelayer medium (soil-litter-air) and accounting for reflection of the microwave intensity at the soil-litter and litter-air interfaces. Detailed equations can be found in (Ulaby et al. [21]). This model will be referred to as the "incoherent" model in the following, since coherent effects are neglected in that modelling approach;

(ii) a numerical model based on a finite elements method which was developed with the ANSOFT's HFSS (High Frequency Structure Simulator) [16] simulation software. The HFSS radiobrightness model was used to compute the reflectivity and the microwave emissivity of a multilayer system, accounting for the moisture content of its different components. Characteristics of the simulations are given in Figure 3.

A plane wave polarized along the $O Y$ axis with propagation vector $\vec{k}$ in direction $O Z$ is generated. The frequency selective surface allows for the calculation of $S_{11}$ coefficients of the model by describing the relationship between the incoming and outgoing fields of the system. The master and slave faces make it possible to impose conditions of periodicity to model an infinite medium in plane $X Y$. Through this condition the model is free of edge effects. A layered impedance boundary condition makes it possible to simulate a succession of layers of different materials and variable thicknesses. During these simulations convergence criteria were imposed on the scattering matrix, one of which was the variation $\Delta S$ of the elements of the scattering matrix. A value of 0.01 (1\%) was chosen and had to be obtained on 2 consecutive iterations of calculation. This model, which accounts for possible coherent effects during the microwave radiation propagation within the soil and

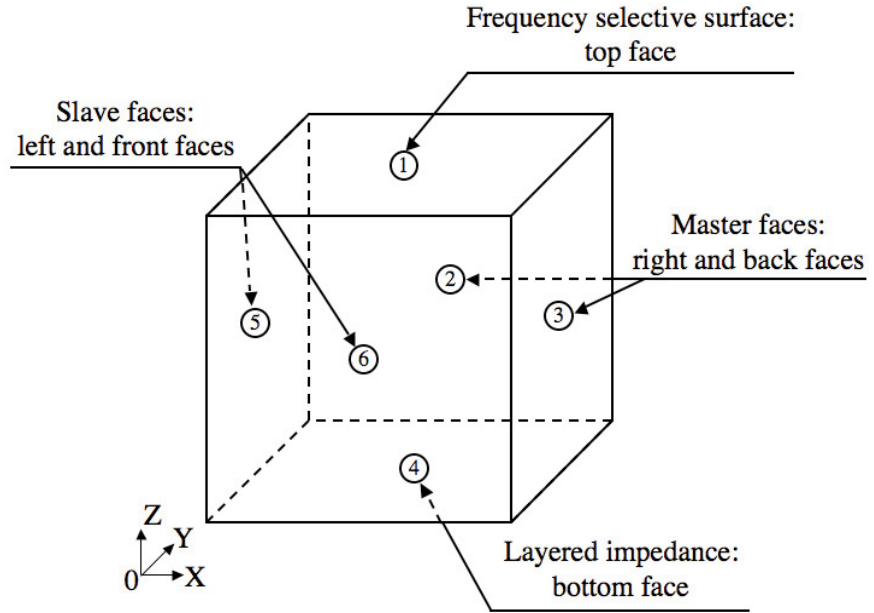

Fig. 3. Presentation of the simulation and the boundary conditions with HFSS.

litter medium, will be referred to as the "coherent" model in the following.

For both models and in order to simplify the analysis, the medium was characterized by plane surfaces at the soil-litter and litter-air interfaces, a uniform structure, and no thermal or moisture gradients. The measured equivalent permittivity of both the soil and the litter media was integrated in the two "coherent" and "incoherent" models so that any phase shifts within the materials themselves were thus neglected. However, using the "coherent" model, phase shifts will occur at the transition between the two media (air-soil and soil-litter). This can involve resonance phenomena. These phenomena are attenuated in a natural litter media due to its heterogeneity and thickness variation. For particular layer sizes, avoiding resonant effects, both the "coherent" and "incoherent" models may produce very similar results (Ulaby et al. [21]).

The main advantage of the "incoherent" model is that it is very simple. However simulations based on that model are not sensitive to coherent effects. Even if coherent effects in natural media are probably attenuated due to (i) heterogeneities within the medium, (ii) roughness characteristics (at the soil-litter and litter-air interfaces) and (iii) litter thickness variations (in relation with rough interfaces), as noted above, these effects may not be negligible and their effects have already been observed from experimental data (Jackson et al., for instance [22]).

Conversely, the coherent model based on the HFSS simulations is much more complex and simulations may be time-consuming for some specific medium configurations. Moreover, for media with plane-interfaces, which are considered in this study, simulations of coherent interference effects are probably over-estimated in comparison to what actually happens in natural media. However, this approach is more powerful than analytical approaches since it can also take into account many characteristics of natural media, such as surface roughness, moisture gradients, thickness variation or the presence of structural heterogeneities. These different variables were not accounted for in the present study but will be the focus of further 
studies for which the advantages of the HFSS model will be very interesting.

In the present study, simulations were mainly based on the coherent modelling approach. However, for some specific cases, simulations using both the "coherent" and "incoherent" models were compared. By comparing simulations based on a model which neglects completely all possible coherent effects (the "incoherent" model) and on a model which overestimates these effects for the specific plane-interface configuration considered in this study (the "coherent" model), we attempted to estimate the possible effects of coherent interferences within natural media.

\subsubsection{Dielectric models}

The model of Dobson [17] is used currently in the forward model ( $L$-MEB) of the SMOS Level 2 algorithm [9]. However, this model was developed from experimental data acquired over a limited range of soil texture and temperature conditions. Recent results by Mironov et al. [20] showed that the Dobson model simulations could not be extrapolated: they may provide poor (or even non physical) results for soils with a large fraction of clay or sand (for fractions of sand larger than about 30\% for instance). However, these soil types represent a very large area at global scale, considering only the semi-arid and arid regions. Recently, a new approach to model the dielectric properties of soils as a function of soil moisture has been proposed and developed by Mironov [23] and will be referred to as the Mironov model. The approach is based on the dielectric spectrum of soil water, which is measured in situ for both free and bound soil water types. It allows the development of a physically based dielectric model for moist soils as the soil water molecules relaxation characteristics can be accurately measured and introduced as physically proven parameters. The Mironov model was used in this study to compute the dielectric properties of soil, considering that this model is more accurate for soils with a large percentage of sand as it is the case for our soil samples (the percentage of sand exceeding generally $\sim 70 \%$ in the Les Landes forest).

\section{Results and discussion}

In this section, the dielectric measurements of the soil and litter samples are analysed as a function of their moisture content. In a preliminary step of the current study, the possible perturbing effects of the water diffusion in the soil and vegetation samples were investigated before further detailed measurements were made. Then, an analysis of the measurement errors was carried out in order to determine the uncertainties associated with the estimation of $\varepsilon^{\prime}$ and $\varepsilon^{\prime \prime}$ due to the sample heterogeneity.

\subsection{Influence of water diffusion in the sample}

The quantity of water injected into the sample was fixed and the sample was also weighed after the measurement

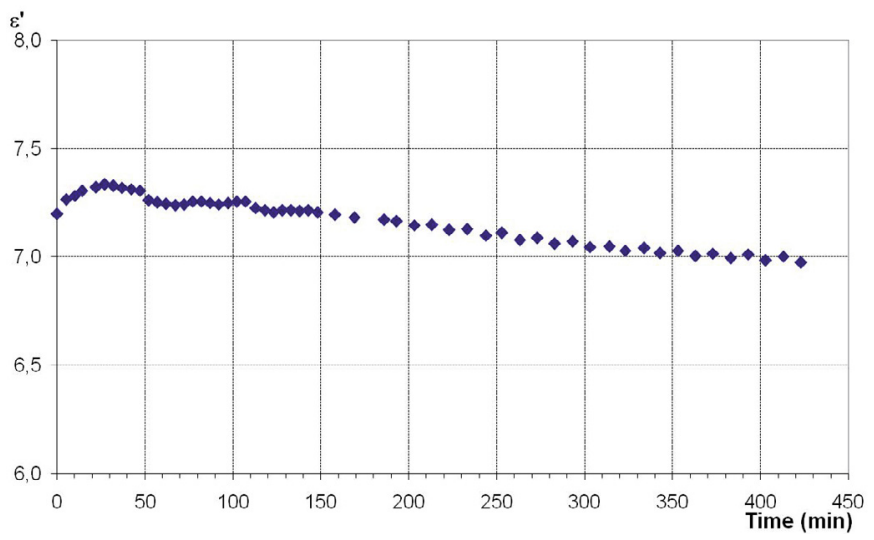

(a)

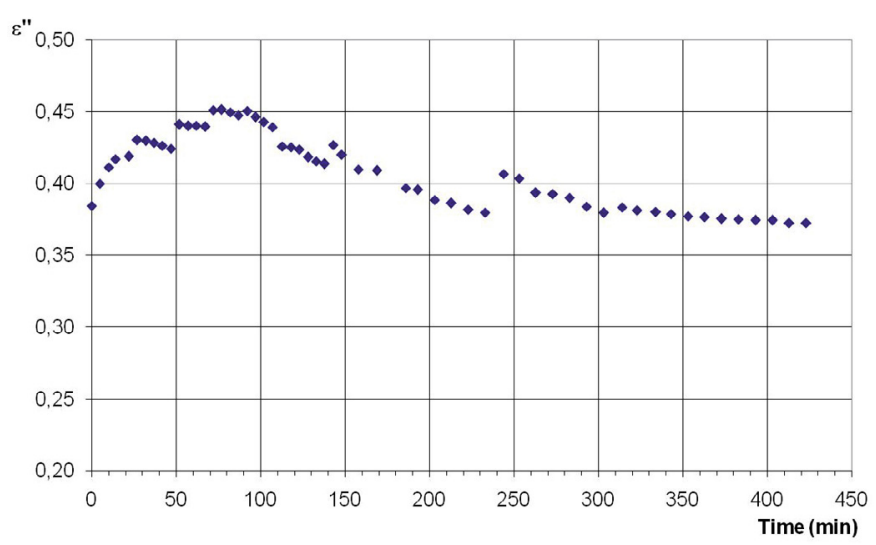

(b)

Fig. 4. Time variations in the measurement of $\varepsilon^{\prime}$ (a) and $\varepsilon^{\prime \prime}$ (b) after water spraying over the soil sample.

to compute its water content. In this way, it was possible to verify that the sample did not dry out during the whole measurement period. The injection of a fixed quantity of water produced a variation of the water content in the sample. However, water diffusion from the surface to the whole volume of the sample may have a significant effect on the measurements. A specific study was carried out to investigate this effect for the soil and litter material. As an illustration, the time variation in the measured permittivity after spraying water over a sample, for a volumetric moisture content of $15.54 \%$ (see Eq. (7)) is shown in Figures $4 a-4 b$.

From these two figures, it is can be seen that the measured values undergo a transitional stage whose duration is $\sim 300 \mathrm{~min}$. After this delay, the measured values are almost stable, as seen in Table 1.

The same procedure was used for litter and results are illustrated for a sample of $16.9 \mathrm{~g}$ with a gravimetric moisture content of $44.95 \%$ in Figures $5 \mathrm{a}-5 \mathrm{~b}$.

An analysis similar to that illustrated in Figures $4 \mathrm{a}-$ $4 \mathrm{~b}$ and $5 \mathrm{a}-5 \mathrm{~b}$ was made for several specific soil and litter samples. From these measurements and the results of the measured standard deviations (presented in Tab. 2), the time we must wait between each measurement 
Table 1. Summary of the results within the soil samples.

\begin{tabular}{ccccc}
\hline & & $\begin{array}{c}\text { Average } \\
\text { value }\end{array}$ & $\begin{array}{c}\text { Standard } \\
\text { deviation }\end{array}$ & $\begin{array}{c}\text { Error on the } \\
\text { average value }(\%)\end{array}$ \\
\hline Based on the entire & $\varepsilon^{\prime}$ & 7.16 & 0.122 & $1.71 \%$ \\
experiment & $\varepsilon^{\prime \prime}$ & 0.41 & 0.029 & $7.14 \%$ \\
\hline Based on measurements & $\varepsilon^{\prime}$ & 7.02 & 0.023 & $0.33 \%$ \\
from 300 mn to the end & $\varepsilon^{\prime \prime}$ & 0.38 & 0.003 & $0.91 \%$ \\
\hline
\end{tabular}

Table 2. Summary of the results within a litter samples.

\begin{tabular}{ccccc}
\hline & & $\begin{array}{c}\text { Average } \\
\text { value }\end{array}$ & $\begin{array}{c}\text { Standard } \\
\text { deviation }\end{array}$ & $\begin{array}{c}\text { Percentage deviation based } \\
\text { on the average value }(\%)\end{array}$ \\
\hline Based on the entire & $\varepsilon^{\prime}$ & 2.58 & 0.026 & $1.00 \%$ \\
experiment & $\varepsilon^{\prime \prime}$ & 0.22 & 0.006 & $2.74 \%$ \\
\hline Based on measurements & $\varepsilon^{\prime}$ & 2.60 & 0.003 & $0.13 \%$ \\
from 200 mn to the end & $\varepsilon^{\prime \prime}$ & 0.22 & 0.003 & $1.24 \%$ \\
\hline
\end{tabular}

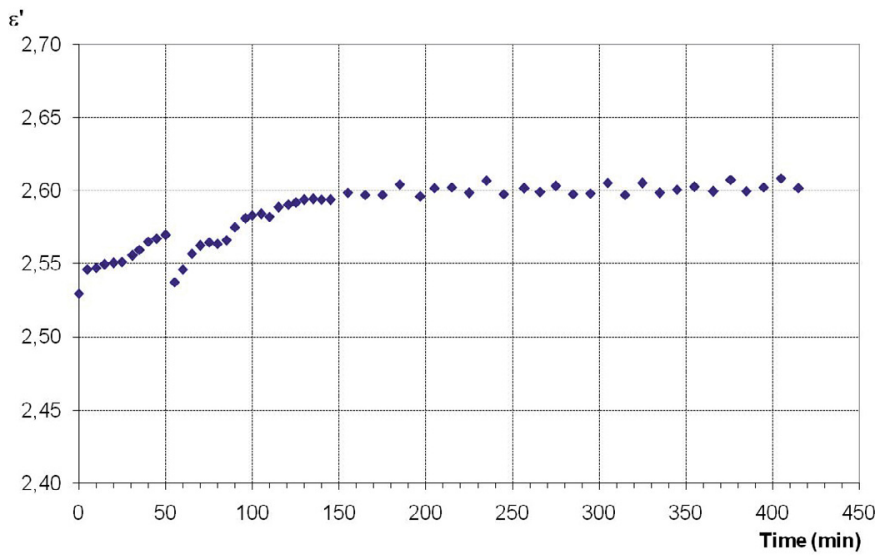

(a)

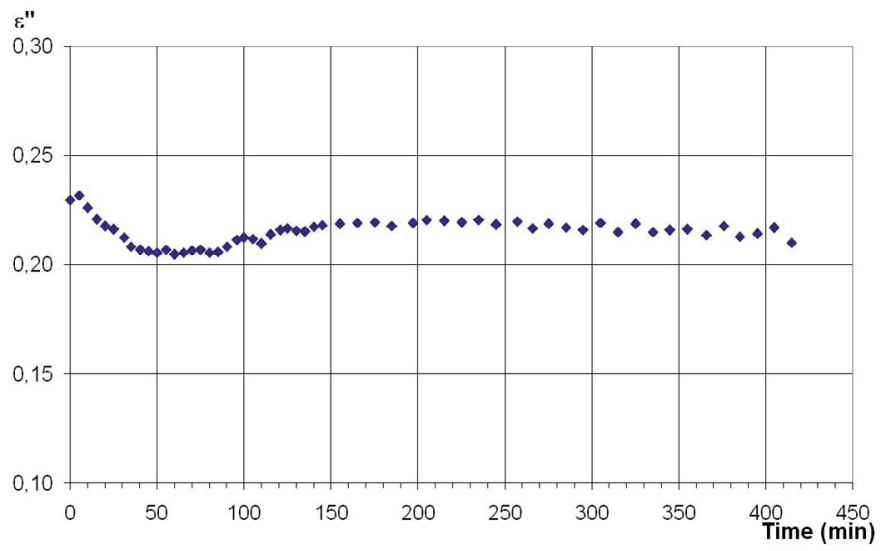

(b)

Fig. 5. Time variations in the measurement of $\varepsilon^{\prime}$ (a) and $\varepsilon^{\prime \prime}$ (b) after water spraying over the litter sample.

was estimated. The analysis showed that a time of about $300 \mathrm{~min}$ for soil and $200 \mathrm{~min}$ for litter was necessary before a stabilization of the measured values can be obtained (see Figs. 4 and 5). This stabilized value, obtained af- ter diffusion processes within the sample were almost finished, was considered as the best estimate of the sample permittivity. This preliminary study highlights a transitory phenomenon in the measurements. It shows that water absorption is not instantaneous and will thus influence the sample permittivity value which is monitored by the continuous measurements. It is therefore necessary to wait for a sufficiently long time until the resulting error is small enough. It is likely that such transitory phenomena may have an effect on the microwave signature of the soil-litter system at the beginning of rainfall events in the real environment.

Under natural conditions, volumetric soil moisture usually varies between $0 \%$ and $40 \%$ and saturation values are generally close to $50 \%$ [17]. For this reason the measurements were limited to the $0-40 \% S M$ range. Based on the preliminary study described above, all further measurements were carried out according to the following protocol for the soil samples:

1) wave guide calibration;

2) measurement of the sample dry weight;

3) introduction of a given quantity of water; weighing of the sample; "instantaneous" measurement of the permittivity (the sample was left for 2 min between the introduction of the water and the "instantaneous" measurement);

4) waiting for $300 \mathrm{~min}$ (for soil);

5) waveguide measurement; weighing of the sample.

Steps $3-5$ were carried out for three soil moisture contents: $12.5 \%, 25 \%$ and $37.6 \%$. The results are presented in Figures $6 \mathrm{a}-6 \mathrm{~b}$. A significant increase in both $\varepsilon^{\prime}$ and $\varepsilon^{\prime \prime}$ with soil moisture can be seen in these figures.

Contrary to the soil, the litter remains wet for a long time and is therefore rarely completely dry in forests. Its gravimetric moisture generally varies between $15 \%$ and $80 \%$, which is the range of litter moisture which will be considered in this study.

The measurements were carried out for litter using a similar protocol to that described above for the soil, but with a waiting period (step 4) of 200 min. Steps $3-5$ were 


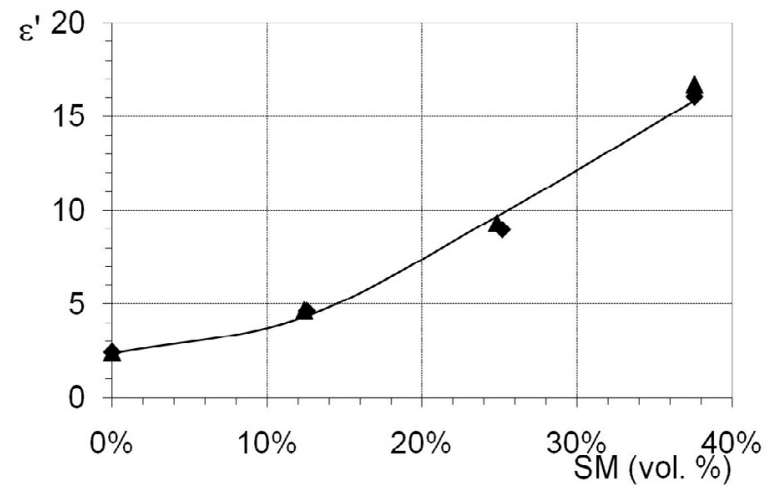

(a)

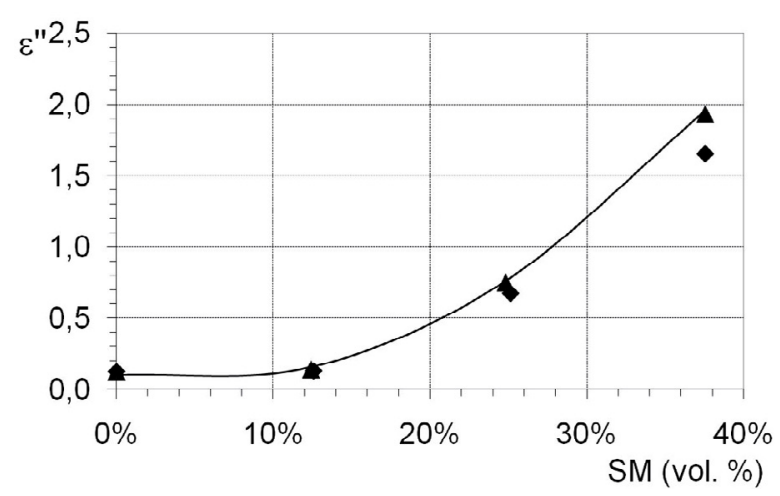

(b)

Fig. 6. (a) Real part of the soil permittivity, (b) imaginary part of the soil permittivity. instantaneous measurements, $\Delta$ measurements after waiting $300 \mathrm{~min}$, - fitted curve.

carried out for five litter moisture contents: $16.6 \%, 31.7 \%$, $44.5 \%, 57.3 \%$ and $66.8 \%$.

Results are given in Figures $7 \mathrm{a}-7 \mathrm{~b}$. As in the case of soil, a significant increase in both $\varepsilon^{\prime}$ and $\varepsilon^{\prime \prime}$ with moisture content can be seen in the figures for litter. A difference between the two types of measurements (instantaneous and after a waiting time of $200 \mathrm{~min}$ ) is observed mainly for $\varepsilon^{\prime}$ at rather high moisture contents.

According to the measurements, the relationship between permittivity and moisture content for the soil and the litter media was approximated by the following analytical formulas (see Figs. 6a-6b and 7a-7b):

for soil:

$$
\begin{aligned}
& \varepsilon_{\text {soil }}^{\prime}=6.5 \tanh (8(S M-0.26))+6.5 S M+8.67 \\
& \varepsilon_{\text {soil }}^{\prime \prime}=\tanh (12(S M-0.28))+0.1 S M+1.1
\end{aligned}
$$

for litter:

$$
\begin{aligned}
& \varepsilon_{\text {litter }}^{\prime}=2.3 \tanh (8(L M-0.65))+5.8 L M+4.1 \\
& \varepsilon_{\text {litter }}^{\prime \prime}=1.25 \tanh (18(L M-0.63))+1.35
\end{aligned}
$$

where tanh is the hyperbolic tangent; $S M$ the volumetric soil moisture $\left(\mathrm{m}^{3} / \mathrm{m}^{3}\right) ; L M$ the gravimetric litter moisture $(\mathrm{kg} / \mathrm{kg})$.

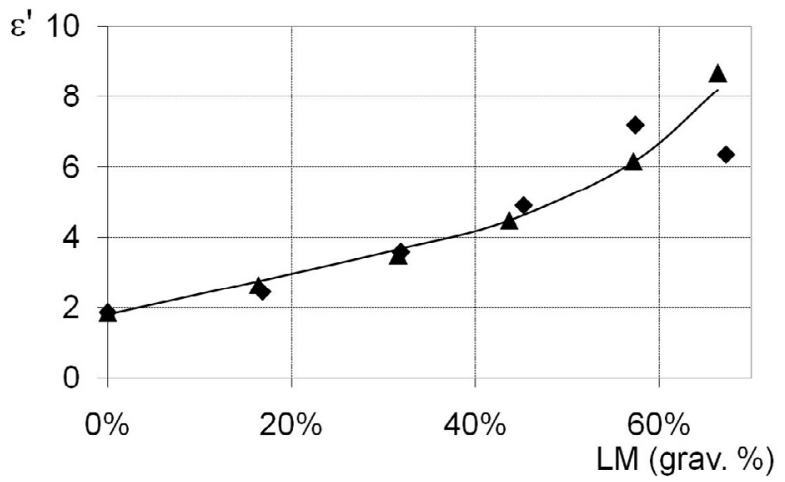

(a)

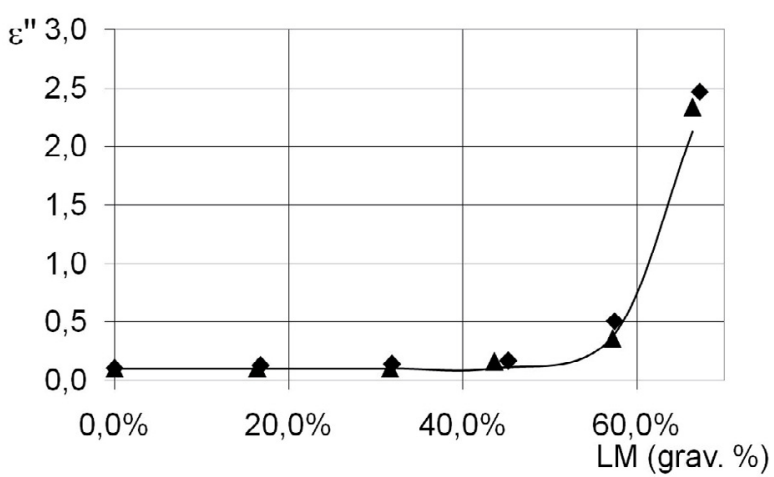

(b)

Fig. 7. (a) Real part of the litter permittivity, (b) imaginary part of the litter permittivity. instantaneous measurements, $\Delta$ measurements after waiting $200 \mathrm{~min}$, - fitted curve.

These equations will be used in the following to compute the soil and litter permittivity values as input data for the "coherent" and "incoherent" model simulations.

For soil, the measurements were compared with simulations made using the Mironov soil dielectric model [20] which computes the soil dielectric constant over a wide range of soil moisture, texture and mineral content. The results of this comparison are given in Figures $8 \mathrm{a}-8 \mathrm{~b}$. We used two values for sand percentage (70 and 80\%) as inputs for the dielectric model corresponding to values generally found in soil samples collected at the Bray site [5]. A good agreement was obtained between measured data and simulations for sand percentage of $70 \%$ for the real part of soil permittivity (Fig. 8a), while the simulations of the imaginary part of the permittivity are similar for both sand contents (70 or $80 \%$ ) and are larger by about $30 \%$ than the measured data. Possible explanations for the discrepancies between measured and simulated values could be related to the effects of the material density (within the measurement cell it was difficult to reproduce exactly the compactness of the original material which was sampled in situ), heterogeneities in the sample which are not accounted for by the Mironov model and measurement errors. To improve our knowledge of these different aspects, an evaluation of the effects of the measurement errors and 


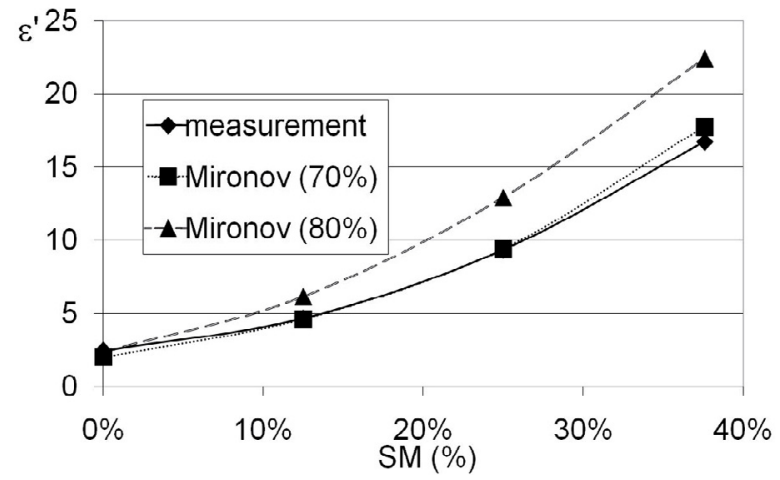

(a)

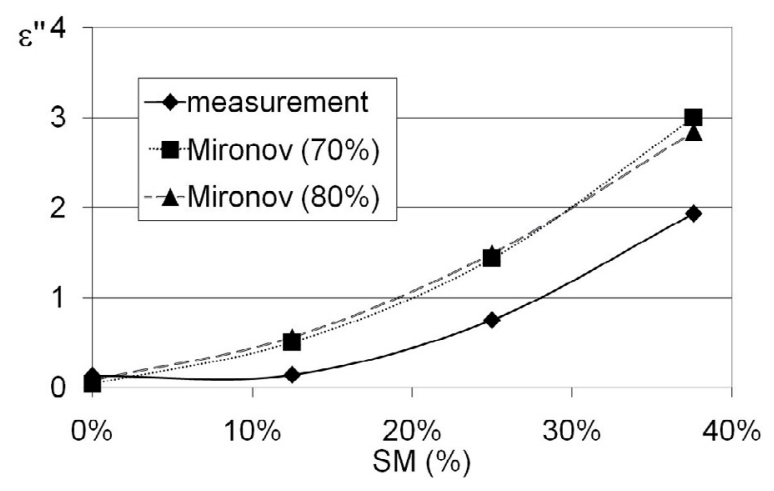

(b)

Fig. 8. (a) Real part of the soil permittivity vs. Mironov model. (b) Imaginary part of the soil permittivity vs. Mironov model.

sample heterogeneity on the measured permittivity values was carried out. It is given in the following paragraph.

\subsection{Measurement errors}

Errors occurring during the measurement procedure of the soil and litter permittivity may have several causes. They are due to the weighing of the sample and support, to the methods of measurement and calculation of the permittivity, and to the sample heterogeneity. These different types of errors are analysed in the following sections.

\subsubsection{Instrumental errors}

As for the uncertainties due to the measurement method, the calibration of the network analyzer corrects for some imperfections of the system but other errors still remain. This kind of measurement uncertainty is random, intrinsic to the apparatus and is due to phenomena such as errors in the measurement of the standards, measurement noise (interfering signals), various drifts (thermics, atmospheric, electronic) or connections.

Other uncertainties related to the method of calculation are due to rounding approximations during numerical calculations. These two types of errors are difficult to quantify, nevertheless, we estimate their influence on the measurement results to be approximately $5 \%$ [18].

\subsubsection{Calculation errors in the sample weighing}

The error in the calculation of moisture is mainly due to errors in sample weighing. The standard deviation of the measured values is $\pm 0.05 \mathrm{~g}$.

The maximum uncertainty of a function $y$ with several variables $x, z$ is calculated in the following way:

$$
\left\{\begin{array}{l}
y=f(x, z) \\
\Delta y=\left|\frac{\partial f}{\partial x}\right| \Delta x+\left|\frac{\partial f}{\partial z}\right| \Delta z .
\end{array}\right.
$$

It then follows from equation (7) that:

$$
\Delta S M=\left|\frac{S M}{W_{W}^{S}}\right| \Delta W_{W}^{S}+\left|\frac{S M}{W_{D r y}^{S}}\right| \Delta W_{D r y}^{S}
$$

and from equation (8) that:

$$
\begin{aligned}
\Delta L M=\left|\frac{L M(1-L M)}{W_{W}^{L}}\right| \Delta W_{W}^{L} \\
+\left|\frac{L M(1-L M)}{W_{D r y}^{L}}\right| \Delta W_{D r y}^{L} .
\end{aligned}
$$

Taking into account the measurements considered in this study, we obtain the following ranges for each variable:

$$
\begin{gathered}
\text { Soil : }\left\{\begin{array}{l}
0 \leqslant S M \leqslant 0.30 \\
0 \leqslant W_{W}^{S} \leqslant 78.50 \mathrm{~g} \\
0 \leqslant W_{D r y}^{S} \leqslant 23.60 \mathrm{~g}
\end{array}\right. \\
\text { Litter : }\left\{\begin{array}{l}
0 \leqslant L M \leqslant 0.66 \\
0 \leqslant W_{W}^{L} \leqslant 34.10 \mathrm{~g} . \\
0 \leqslant W_{D r y}^{L} \leqslant 17.30 \mathrm{~g}
\end{array}\right.
\end{gathered}
$$

Using the above values together with equations (16) and (17) we assume an error of $\pm 0.10 \mathrm{~g}$ on the weights, because the water and dry weights are calculated, respectively, by differences between the wet and dry weights, and between the weight of the sample and the support and the weight of the support alone. For soil and litter, this results in errors in soil and litter moisture of $\pm 0.17 \%$ and $\pm 0.58 \%$, respectively.

\subsubsection{Sample heterogeneity}

As an attempt to account for the heterogeneity of the medium in our results, we tested five different types of measurements: 
Table 3. Average values and standard deviations of the measured permittivity for specific moisture contents of the litter or soil sample.

\begin{tabular}{cccc}
\hline & Moisture & Average value & Standard deviation \\
\hline$\varepsilon^{\prime}$ soil & $40 \%$ & 19.66 & 0.4 \\
$\varepsilon^{\prime \prime}$ soil & $40 \%$ & 2.95 & 0.14 \\
\hline$\varepsilon^{\prime}$ litter & $16.6 \%$ & 2.53 & 0.1 \\
$\varepsilon^{\prime \prime}$ litter & $16.6 \%$ & 0.16 & 0.02 \\
\hline
\end{tabular}

- No. 0: initial measurement;

- No. 1: the sample is taken out of the guide and reinstalled in the guide before measuring the permittivity;

- No. 2: the sample is taken out of the guide, turned $180^{\circ}$ and positioned back in the guide before measuring the permittivity;

- No. 3: the sample is modified (the material is mixed inside the guide) before measuring the permittivity;

- No. 4: the sample is modified, taken out of the guide and reinstalled in the guide before measuring the permittivity;

- No. 5: the sample is mixed, taken out of the guide, turned $180^{\circ}$ and reinstalled in the guide before measuring the permittivity.

The tests were made for various moisture conditions. Examples of the results obtained for one moisture content for the litter and for soil samples are given in Table 3 .

Taking the results of all moisture conditions together, it was possible to estimate the standard deviation due to the heterogeneity of the medium. This value was then considered in the final result through the form of a range around each measured value of permittivity.

\subsection{Permittivity ranges}

The various errors or measurement variations presented above led to the definition of a range of permittivity which changes according to the moisture content of the considered medium, instead of a sole curve describing the permittivity changes as a function of the sample moisture content. Two ranges were defined, the first one was called the range of possible permittivities and the second the corrected range of permittivity. The first range includes the observed measurement fluctuations (accounting for the heterogeneity of the medium) while the second additionally includes the measurement errors. The first range is obtained by adding the estimated standard deviation to the measured values (see Sect. 3.2.3). Then the second range is obtained by adding the instrumental error (see Sect. 3.2.1) and the error due to the sample weighing procedure (see Sect. 3.2.2) to this first range. These results are presented in Figures 9 and 10. In these figures, the dashed lines are the boundaries of the range of possible permittivities and the continuous lines represent the permittivity range including all the listed errors. The measurements were done at different moisture contents (see

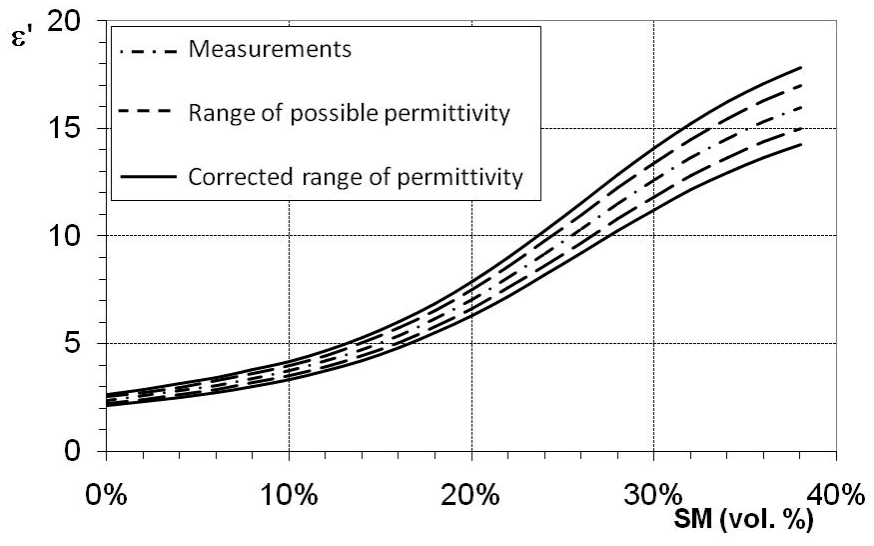

(a)

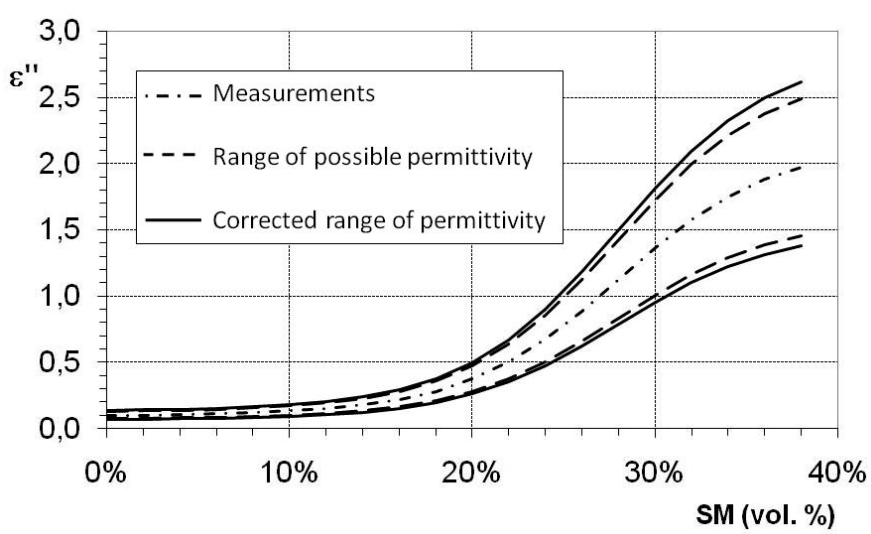

(b)

Fig. 9. (a) Range of the real part of the soil permittivity. (b) Range of the real and imaginary part of the soil permittivity.

previous study in Sect. 3.1) and the curves were obtained by interpolating computed values between two successive moisture contents.

Now that ranges of permittivity have been computed taking into account measurement errors and sample heterogeneities, measured values were again compared against simulated values using the Mironov soil dielectric model [20] in Figures $11 \mathrm{a}-11 \mathrm{~b}$. It can be seen that the estimated range of the measured values of $\varepsilon^{\prime}$ are in good agreement with the Mironov simulations using a sand fraction of $70 \%$ (Fig. 11a). Also, it can be seen (Fig. 11b) that the higher limit of the range of measured values of $\varepsilon^{\prime \prime}$ is relatively close to the Mironov simulations using a sand fraction of $70 \%$ or $80 \%$. Differences between measured and simulated values of $\varepsilon^{\prime \prime}$ could be due to the effects of soil heterogeneities (for instance the presence of organic matter) and density (the sample compactness may affect the value of the measured $\left.\varepsilon^{\prime \prime}\right)$, which are not taken into account in the simulations. 


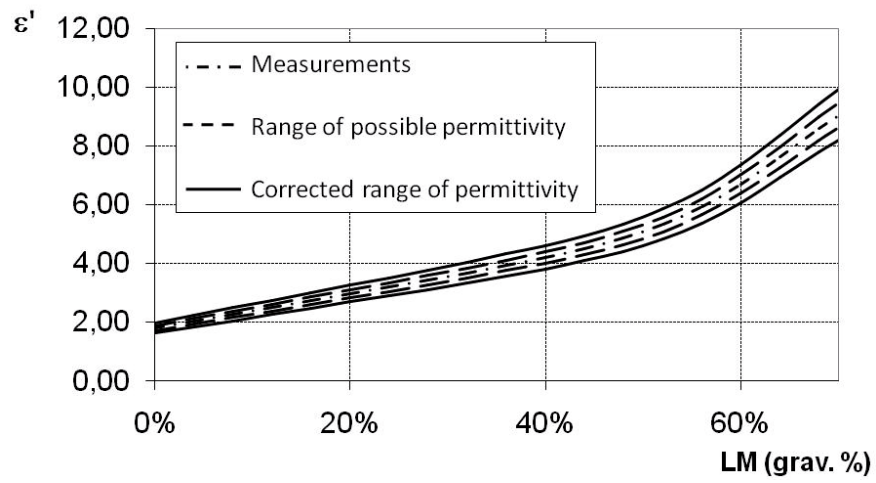

(a)

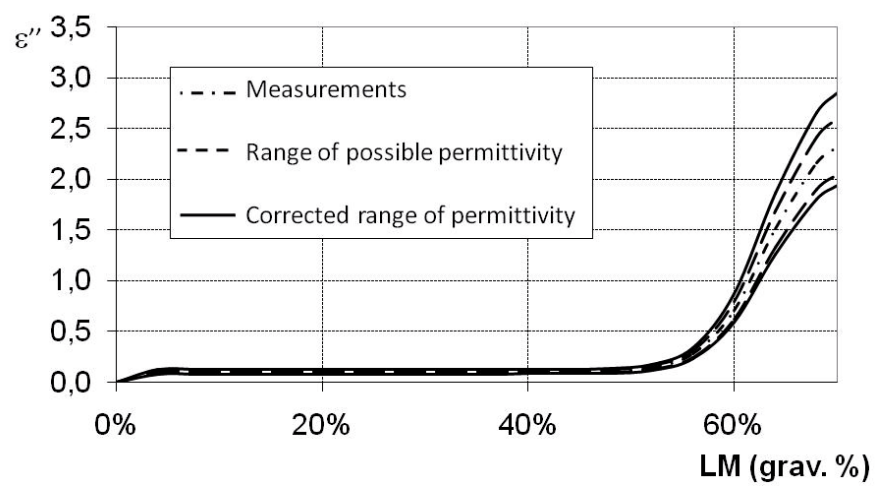

(b)

Fig. 10. (a) Range of the real part of the litter permittivity. (b) Range of the imaginary part of the litter permittivity.

\subsection{Simulation of the "Les Landes" surface microwave emissivity}

In this section, we investigated the effect of litter on the surface microwave emissivity. Simulations were made with the coherent and incoherent models for soil and litter conditions corresponding to those of the Les Landes forest. We used the dielectric measurements made over soil and litter and described in the previous section. The simulations were made for a system of one or two layers (soil or soil-litter) whose thicknesses is semi-infinite for soil and varies between 3 and $10 \mathrm{~cm}$ for litter. Ground measurements made at the same test site during a 6 month period and for varying wetness conditions made it possible to compute an approximate relationship between the volumetric moisture of the soil $(S M)$ and the gravimetric moisture of litter $(L M)[5]$ :

$$
L M(\text { grav. \% })=2.7201 S M(\text { vol. \% })-8.6223 \text {. }
$$

This relationship was used in the current study and changes in $L M$ were directly derived from changes in $S M$. In the simulations, the volumetric soil moisture content varied between $0 \%$ and $30 \%$, resulting in litter moisture contents between $0 \%$ and $73 \%$. We used both the coherent and incoherent models, defined in the previous sections, to compute the emissivity of the soil-litter system. The re-

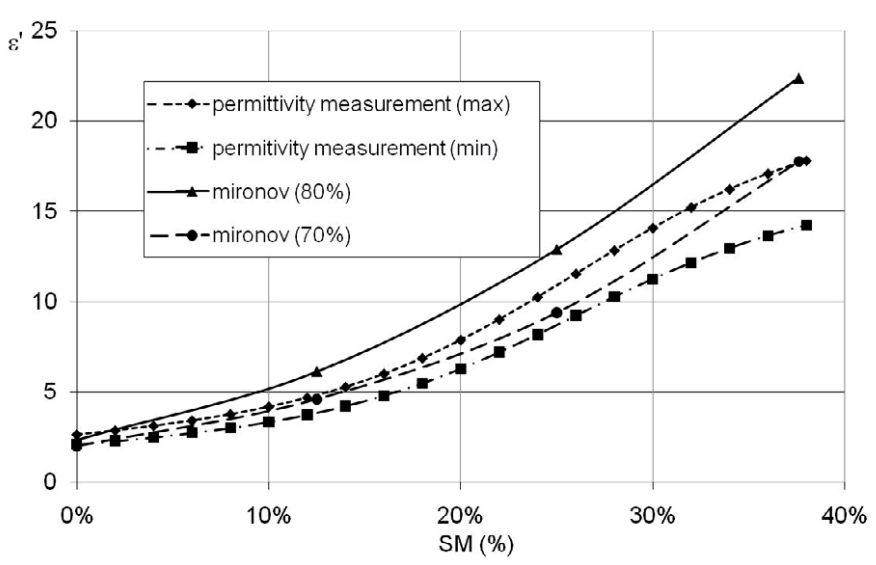

(a)

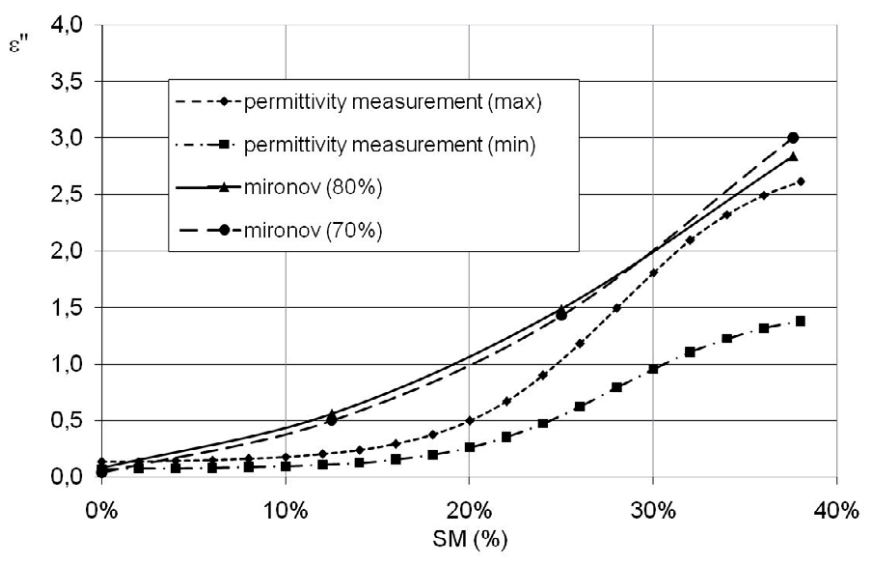

(b)

Fig. 11. (a) Range of the real part of the soil permittivity vs. Mironov model. (b) Range of the imaginary part of the soil permittivity vs. Mironov model.

sulting effects of the litter layer thickness on the emissivity of the soil-litter system are illustrated in Figures 12a-12b.

For both models, it can be seen in these figures that the litter characteristics, in terms of moisture content and thickness, have a strong influence on the emissivity of the soil-litter system. The emissivity of a soil surface overlaid by a litter layer is always higher than that of a bare soil, considering both model simulations and all possible configurations in terms of soil moisture or litter thickness. When the litter thickness increases, two inverse effects affect the soil-litter emission: (i) a decrease in soil contribution due to an increased attenuation of the soil emission by the litter layer and (ii) an increase in litter emission due to the increase in litter biomass [9]. It seems that the effects of litter self-emission are the most important so that the emissivity of the soil-litter system is larger than that of the soil surface alone.

However, except for this result, predictions given by both models are generally quite different. Using the incoherent model, simulations for a litter thickness of 3,6 or $10 \mathrm{~cm}$ produced almost the same emissivity values and 


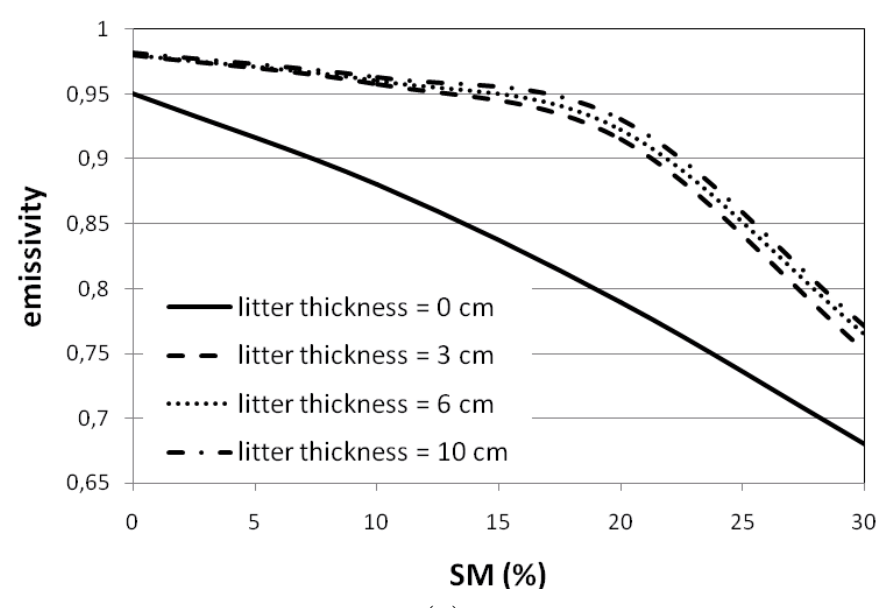

(a)

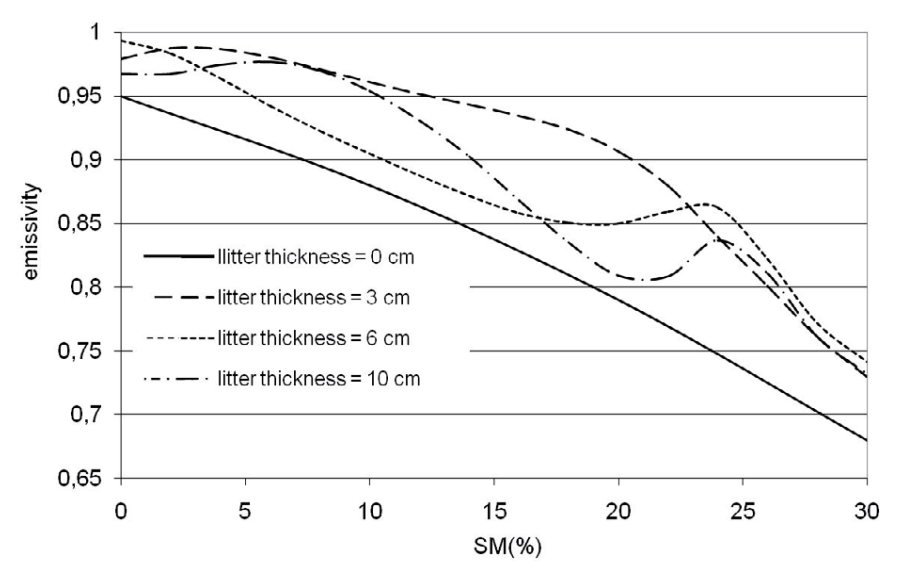

(b)

Fig. 12. Emissivity of the soil-litter system computed as a function of soil moisture for several values of the litter thickness $(0,3,6$ and $10 \mathrm{~cm})$ using the incoherent (a) and coherent (b) model.

emissivity is found to be a decreasing function of soil moisture (and litter moisture through Eq. (18)).

Conversely, using the coherent model, the emissivity is not a decreasing or increasing function of the litter thickness or moisture content. For instance, for $S M \leqslant 20 \%$, the emissivity of the soil-litter system for a litter thickness of $3 \mathrm{~cm}$ is predicted to be larger than for a litter thickness of 6 or $10 \mathrm{~cm}$. Also, small "peaks" in the function relating emissivity to $S M$ can be seen for values of $S M$ close to $25 \%$ (for a litter thickness of 6 or $10 \mathrm{~cm}$ ). It is likely that all these effects obtained with the coherent model are due to coherent interference effects. The pattern of oscillation corresponding that these "peaks" is similar to that predicted by coherent radiative transfer models for a layered media with sharp dielectric boundaries [21,22], such as the soil-litter and litter-air interfaces considered in this study.

It is likely that such coherent interference effects are not so marked in natural environment as such "sharp" interfaces do not exist, in relation with the natural spatial variations in litter characteristics (in terms of thickness, moisture content, composition, structure, etc.). However they might not be completely absent, as predicted by the incoherent model, which neglects all possible coherent interference effects.

In a second step the soil-litter emissivity was simulated accounting for heterogeneities of the litter and soil media and for uncertainties in the measurements of the litter and soil dielectric constants as shown in Figures 9 and 10. To do this, each measured permittivity range was introduced into the models (coherent and incoherent) and then the resulting emissivity range was computed. A value of $3 \mathrm{~cm}$ for the litter layer thickness was used as this value is close to the actual (spatially averaged) value measured in the Les Landes forest. The resulting soil-litter emissivity trend as a function of $S M$ is given in Figure 13.

In this figure, the ranges of computed emissivity for the soil-litter (grey zone) and bare soil (black zone) system are presented. The large effects of the litter on the global soil-litter emissivity can be noted. Errors on litter and soil permittivity measurements lead to variations in the emissivity calculations (grey zone) up to $\sim 0.025$ (at $S M=$ $30 \%$ ), which corresponds to an error of $3.7 \%$ relatively to the value of the soil-litter emissivity $(\sim 0.68)$. This value is small in comparison with effects of litter overlying the bare soil surface.

For instance, the presence of the litter layer (for $S M=$ $30 \%$ and a $3 \mathrm{~cm}$ litter layer thickness) lead to an increase in emissivity of about 0.09 , compared to the value for the bare soil surface. This increase corresponds to a variation of $6.5 \%$ relatively to the average value for this soil moisture ( $S M=30 \%$ ) of the calculated soil and soil-litter emissivity. In the worst case (minimum value of soil emissivity and maximum value of the soil-litter emissivity computed for the same soil moisture) this increase in emissivity due to the presence of litter may reach $\sim 0.153$, corresponding to a relative variation of emissivity of $\sim 20.4 \%(S M \approx 22 \%)$.

\section{Summary and conclusion}

In this study, a technique for measuring the permittivity of soil and litter media was investigated. This technique was based on a rectangular waveguide containing the soil or litter sample and takes into account the heterogeneity of natural media. In this study, the measurements were carried out over soil and litter samples which were collected in a coniferous forest in the region of Les Landes. The measurements of the soil and litter dielectric constants were used in two models (an incoherent and a coherent numerical model) to simulate the microwave emissivity of the soil-litter system. Using a specific relationship between litter moisture and soil moisture, estimated from ground measurements over the same forest type these simulations allowed us to evaluate the effects of soil moisture content, litter moisture content and litter thickness on the emissivity of such a system.

It was found that variations in the dielectric constant of the litter and soil media had a strong influence on the resulting soil-litter emissivity. Using the incoherent model, the simulations showed that the soil-litter emissivity generally increases as a function of litter thickness. This trend 


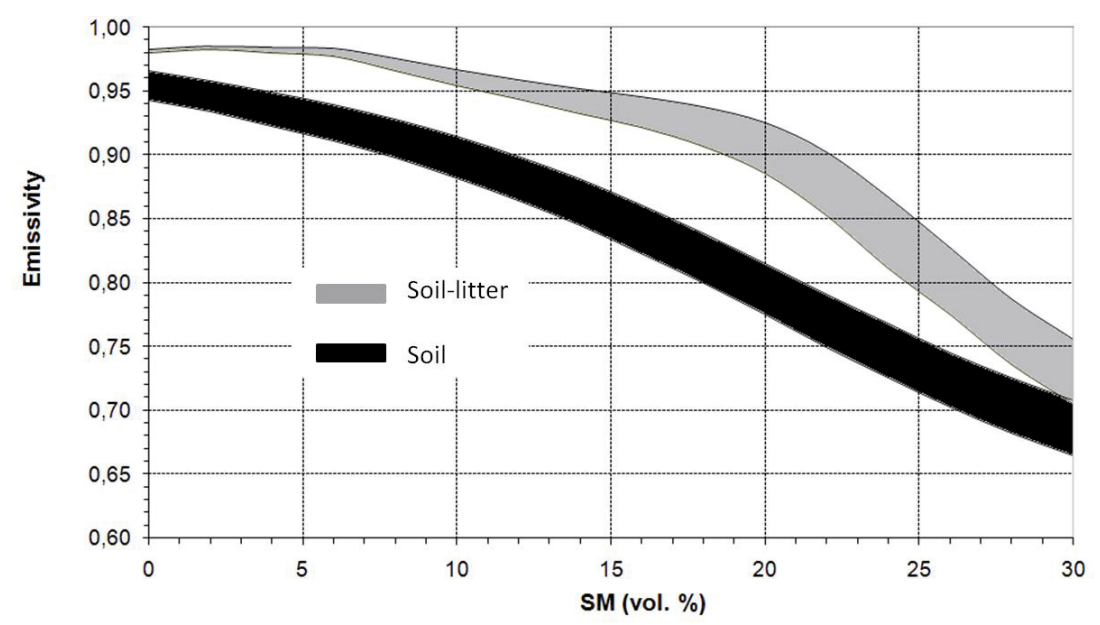

Fig. 13. Soil-litter and bare soil emissivity computed accounting for the permittivity ranges computed in the previous sections - coherent model simulation.

results from two inverse effects that affect the soil-litter emission: (i) a decrease in the soil contribution due to an increased attenuation of the soil emission by the litter layer and (ii) an increase in litter emission due to the increase in litter moisture content. The fact that the emissivity of the soil-litter system generally increases as a function of litter layer thickness indicates that the effects of litter self-emission are important. However, using the coherent model, the soil-litter emissivity was found to be a non monotonous function of litter thickness. This result could be explained by resonance effects parameterized by the ratio of the radiation wavelength (around $20 \mathrm{~cm}$ at $L$ band in free space) and the litter thickness. In particular, the general decreasing form of the relationship relating the emissivity to the soil moisture content $(S M)$ was affected by small peaks close to the value $S M=25 \%$. Considering the disagreements between the simulations based on the two modelling approaches (coherent and incoherent), more studies based on experimental measurements are required to define which modelling approach is best suited to simulate the emissivity of the soil-litter system in natural environment.

In a second step of the study, particular attention was given to measurement errors in the calculation of permittivity and emissivity. Uncertainties in the values of soil-litter emissivity, computed as a function of $S M$, were evaluated. It was found that these uncertainties were relatively low in comparison with the range of emissivity variations due to the effects of soil moisture (between dry and wet surface conditions) or litter thickness (between 3 and $10 \mathrm{~cm}$ in this study).

All the simulations carried out in this study were done for uniform conditions of the soil-litter system, i.e. assuming no heterogeneities in the soil structure, no soil or litter roughness (the soil-litter and litter-air interfaces were assumed to be plane) and no moisture or thermal gradients in the soil and litter media. Future studies will consider more realistic conditions of soil and litter heterogeneity. In the present study a uniform litter layer was considered (of 3,6 or $10 \mathrm{~cm}$ thickness) although it was shown that the thickness of this layer significantly influenced the emissivity of the soil-litter system. In the natural environment, changes in the litter thickness from $\sim 1 \mathrm{~cm}$ to $\sim 15 \mathrm{~cm}$ generally occurred in the forest at small spatial scales $(\sim 1 \mathrm{~m})$ due to natural changes in soil roughness, topography, tree density, etc. Therefore, further studies will consist of studying long-term variations of the soil-litter emission and will account for a variety of conditions in terms of thermal and moisture gradients within the soil and litter layers, as well as specific effects such as frost.

\section{References}

1. ESA website: http://www.esa.int/esaLP/LPsmos.html

2. CNES website: http://www.cnes.fr/web/821-smos.php

3. EADS Space website: http://www.space.eads.net/. Link: Press centre/Press documents/Flight Model of the SMOS payload, built by EADS Astrium in Spain, ready for final testing at ESA, January 16, 2007

4. ESA, Algorithm Theoretical Based Document (ATBD), SMOS level 2 processor for soil moisture, Prepared by the Expert Support Laboratories (CESBIO, IPSL-Service d'Aéronomie, INRA-EPHYSE, Reading University, Tor Vergata University, 2006), p. 34, http://www .cesbio.ups-tlse.fr/us/indexsmos.html

5. J.P. Grant, J.P. Wigneron, A.A. Van de Griend, A. Kruszewski, S.S. Søbjærg, N. Skou, Remote Sens. Environ. 109, 10 (2007)

6. T.J. Jackson, T.J. Schmugge, Remote Sens. Environ. 36, 203 (1991)

7. T.J. Schmugge, J.R. Wang, G. Asrar, IEEE Trans. Geosci. Remote Sens. 26, 590 (1988)

8. K.Saleh, J.-P. Wigneron, P. de Rosnay, J.-C. Calvet, M.J. Escorihuela, Y.H. Kerr, P. Waldteufel, Remote Sens. Environ. 101, 127 (2006)

9. J.-P. Wigneron, Y.H. Kerr, P. Waldteufel, K. Saleh, M.J. Escorihuela, P. Richaume, P. Ferrazzoli, P. De Rosnay, R. Gurney, J.-C. Calvet, J.P. Grant, M. Guglielmetti, B. Hornbuckle, C. Mätzler, T. Pellarin, M. Schwank, Remote Sens. Environ. 107, 639 (2007) 
10. J.P. Wigneron, J.-C. Calvet, T. Pellarin, A.A. Van de Griend, M. Berger, P. Ferrazzoli, Remote Sens. Environ. 85, 489 (2003)

11. F.T. Ulaby, R.K. Moore, A.K. Fung, Microwave remote sensing: Active and passive, From theory to Applications, Vol. III (Artech House Inc., 1986), p. 2099

12. W.B. Weir, Proc. IEEE 62, 33 (1974)

13. F. Demontoux, G. Ruffié, J.P. Wigneron, Amélioration de l'étude de l'humidité de sols par radiométrie. Caractérisation et modélisation diélectriques de profils géologiques, Journées Nationales Microondes, Nantes, 2005

14. P. Sabouroux, Ph.D. thesis, University of Bordeaux 1, 1992, pp. $34-36$

15. F.T. Ulaby, R.K. Moore, A.K. Fung, Microwave remote sensing: Active and passive, Microwave Remote Sensing, Fundamentals and Radiometry, Vol. I (Addison-Wesley Publishing Company, 1981), pp. 238-244

16. Ansoft website: http://www.ansoft.com. HFSS online help, assigning boundaries

17. M.C. Dobson, F.T. Ulaby, M.T. Hallikainen, M.A. ElRayes, IEEE Trans. Geosci. Remote Sens. 23, 44 (1985)

18. Operation Manual "Vector Network Analyser Model 373XX" Appendix C, Performance Specifications P/N 10410-00183 (Anritsu CO, Nov. 1998 (C)1996)
19. J.P. Wigneron, Doctorat Université P. Sabatier (Toulouse, 1993), pp. 17-20

20. V.L. Mironov, M. Craig Dobson, V.H. Kaupp, S.A. Koramov, V.N. Kleshchenko, IEEE Trans. Geosci. Remote Sens. 42, 773 (2004)

21. F.T. Ulaby, R.K. Moore, A.K. Fung, Microwave remote sensing: Active and passive, From Theory to applications, Vol. III (Addison-Wesley Publishing Company, 1981), pp. $1524-1531$

22. T.J. Jackson, T.J. Schmugge, P.E. O'Neill, M.B. Parlange, IEEE Trans. Geosci. Remote Sens. 36, 1376 (1998)

23. V.L. Mironov et al., Physically and Mineralogically Based Spectroscopic Dielectric Model for Moist Soils, IEEE Trans. Geosci. Remote Sens. (accepted)

24. M. Guglielmetti, M. Schwank, C. Mätzler, C. Oberdörster, J. Vanderborght, H. Flühler, IEEE Trans. Geosci. Remote Sens. 46, 3 (2008)

25. M. Schwank, M. Guglielmetti, C. Mätzler, H. Flühler, IEEE Trans. Geosci. Remote Sens. 46, 7 (2008) 